\title{
SOMALI HEALTH ACTION JOURNAL - Iskaashi wada shaqeyn oo loogu talagalay Cilmi-baarista Caafimaadka iyo Horumarinta
}

$\mathrm{K}^{\prime}$ u soo dhowow "Somali Health Action Journal" (SHAJ), oo ah joornaal $\mathrm{u}$ furan dhammaan akhristayaasha iyadoo loo adeegsanayo internet.

SHAJ waa madal aqoon-wadaag heer qaran iyo mid caalami ah, laguna soo bandhigo mawduucyo muhiiim u ah nidaamka caafimaadka Soomaaliya oo ah dal burburka u jilicsan oo ka soo gudbay colaado saameeyey horumarinta caafimaadka Soomaaliya iyo deegaamo kale oo soo maray xaalado la mid ah.

Ujeedada joornaalku waa inuu $u$ gogol-xaaro isdhexgalka aqoonyahannada Soomaaliyeed iyo hay'adahooda, xirfadlayaasha nidaamka caafimaadka, siyaasad-dajiyeyaasha iyo shuraakada caalamiga ah, si loo horumariyo xalal ku saleysan caddeymo maxalli ah oo loogu talagalay wax ka qabadka dhibaatooyinka caafimaad ee dadka. Himilooyinka Joornaalka waxaa ka mid ah dhisidda iskaashiyo cilmi-baaris caafimaad iyo kobcinta dabeecadaha iyo qiyamka cilmi-baaris, iyada oo diiradda la saarayo baahiyaha caafimaad ee mudnaanta leh. Wuxuu sidoo kale higsanayaa horumarinta dhisidda awoodda cilmi-baarista ee saameysa dhammaan xarumaha waxbarashada caafimaad iyo iskaashiga cilmi baarista ee jaamacadaha.

Fikirka joornaalkani wuxuu ka soo unkamay dhowr seminaar iyo aqoon-iswaydaarsiyo caalami ah oo lagu qabtay Sweden, laga soo bilaabo 2014 iyo wixii ka dambeeyay. Ka qeyb galayaashu waxay isugu jireen wakiillo ka kala socday lix jaamacadood oo soomaali ah, shan jaamacadood oo Sweden ka socda iyo sidoo kale aqoonyahanno qurbajoog soomaaliyeed ah. Ujeeddadu waxay ahayd in dib loo soo nooleeyo barnaamijkii iskaashiga cilmi-baarista ee Soomaaliya iyo Sweden, kaas oo la joojiyay horraantii 90: meeyadii sababo la xiriira colaado sokeeye. Iyada oo diiradda la saarayo caafimaadka iyo caqabadaha lagala kulmay dib u dhiska caafimaadka soomaalida iyo nidaamka daryeelka caafimaad, ayaa wada-hadaladaasi waxay tilmaameen ahamiyadda ay leedahay cilmi-baaris $\mathrm{ku}$ wajahan caafimaadka dadweynaha iyo doorka muhiimka ah oo ay ka qaadan karaan jaamacaduhu dhisidda awoodda fulinta si loo gaaro ujeedadan $[1,2]$. Tani waxay si wada-jir ah ugu horseedday abaabulidda barnaamij tababar cilmi-baaris caafimaad.

Koorsada koowaad waxay awood u siisay dhalinyarada aqoonyahannada ah ee ka socday jaamacadaha soomaalida iyo kuwa ku hawllan daryeelka caafimaadka, inay si guul leh u dhammaystaan, ugana warbixiyaan mashaariicda cilmi-baaris ee ay fuliyeen iyaga oo taageero ka helay lataliyeyaal Soomaali iyo kuwo reer Sweden ah. Maqnaanshaha joornaal maxalli ah oo lagu faafiyo natiijooyinka cilmi-barista awgeed, waxa ilaa iyo hadda lagu daabacay joornaal caalami ah labo ka mid ah warbixinnadaas [3, 4], kuwa kalena dadaalku waa socdaa.

Iyada oo la aqoonsanyahay yaraanta qoraallada cilmibaaris, gaar ahaan kuwa ka yimaada qoraayaal soomaaliyeed ee ku taxalluqa dhibaatooyinka caafimaadka soomaalida, ayaa dhinacyada fulinaya iskaashigani waxay go'aansadeen in ay si wadajir ah u abuuraan madal loogu talagalay in lagu faafiyo cilmi-baarista iyo doodaha siyaasadeed ee la xiriira. Intii lagu guda jiray hawshan qorshaynteeda, waxaa la isku raacay in SHAJ uu yahay hanti ay leeyihiin jaamacadaha Soomaalidu. Hase yeeshee, sababo la taaban karo awgood, waxa si ku meel gaar ah Joornaalka muddada hore marti-gelin-doona Ururka Cilmibaarayaasha Soomaalida iyo kuwa Sweden (Somali Swedish Researchers' Association-SSRA), oo ah hay'ad aan dawli ahayn oo fadhigeedu yahay Sweden. Hindisaha SHAJ waxaa sidoo kale taageeray Wakiilka Soomaaliya ee Ururka Caafimaadka Adduunka.

Bilow ahaan, dhowr maqaal oo cilmi-baarayaal loo idmaday ay qoreen, ayaa diiradda lagu saari doona arrimo caafimaad oo muhiim ah iyo hawlgallo saameeya xaaladda 
caafimaad ee Soomaaliya. Qoraaladaas waxaa ku jiridoona warbixinno laga soo diyaariyey mashaariic socda oo ku saabsan arrimo kala duwan sida caafimaadka dhimirka/maanka, gudniinka/jarista xubin taranka haweenka, istratiijiyado lagu horumarinayo nidaamyada caafimaad ee abuurmay colaadaha kaddib iyo xakamaynta duumada Soomaaliya. Dib-u-eegis loo adeegsanayo habka qoraallada lagu falaanqeeyo ee loo yaqaan "bibliometric review" ayaa iyana qiimeyn doonta cidda daabacday maqaallada ku saabsan arrimaha caafimaadka Soomaaliya ee 40-kii sano ee u dambeeyey.

Waxaa qoraayaasha lagu martiqaadayaa soo gudbinta maqaallo looga doodayo fursadaha siyaasadeed ee loogu talagalay iney horkacaan dib-u-dhiska nidaamka caafimaadka iyo dowrka ay shabakadda cilmi-baaristu ka gelayso u-hawlgalka dib-u-dhiskaas.

Baahida loo qabo faafinta cilmi-baarista ayaa sabab weyn u ah daah-furidda joornaalkan, annaga oo ku rajoweyn in uu dardar gelindoono himilooyinka qaran ee dhaqan-gelinta "Baahinta caalami ee Caafimaadka" (Universal Health Coverage-UHC). UHC iyo Yoolalka Waara ee Horumarinta (Sustainable Development GoalsSDGs), ayaa si aan kala go' lahayn ula xiriira yoolalka caafimaadka ee la higsanayo 2030-ka.

Joornaalku wuxuu cabbiri doonaa fikradaha $\mathrm{ka}$ imanaya in badan oo xirfadlayaasha caafimaadka soomaaliyeed ah iyo hey'adaha tacliinta oo tobanaan sano ku shaqeynayey nidaam caafimaad oo jilicsan iyo awood cilmi-baaris xaddidan. Madasha "digital" ee soo ifbaxday ayaa dhallinyarada aqoonyahanada somaaliyeed iyo shaqaalaha daryeelka caafimaadka siin doonta fursad ay ku helaan macluumaad caafimaad dheeri ah oo weliba caddeyn ku salaysan.

\section{Joornaalka SHAJ waa awood kordhinaysa soo kabashada nidaamka caafimaadka}

Joornaalku wuxuu u hawl-gelidoonaa sida gole diiwaangelin iyo baaris fulinta daryeelka iyo cilmi-baarista caafimaadka, iyada oo waxqabadkaas lala beegsanayo arrimaha mudnaanta leh. Markii la tixgeliyo xaaladda Soomaaliya, waxaa muhiim ah in habka waxqabadka diiradda lagu saaro cudurada culeyska ku haya oo inta badan saameeya dadka saboolka ah iyo kuwa danyarta ah. SHAJ oo kaalinteedu tahay ergayga caafimaadka bulshada, waxay kobcin doontaa oo diiradda saari doontaa arrimaha soo socda:

- Fulinta cilmi-baaris lagu xallinayo culeyska badan ee ka dhasha caqabadaha caafimaad darro, kuwaas oo beegsanaya caafimaadka deegaanka ee mudnaanta leh, iyo in la isku xiro hay'adaha tacliinta sare iyo xarumaha daryeelka caafimaadka dawladda iyo kuwa gaarka loo leeyahay.

- Cilmi-baaris ku saleysan bulshada, oo ah istraatiijiyad muhiim $\mathrm{u}$ ah horumarin wax ku ool ah, oo dhaqan ahaan bulshada khuseeya, lamahuraanne $\mathrm{u}$ ah waxqabadka caafimaad iyo horumarinta doorka iyo mas'uuliyadaha hay'adaha caafimaadka, si ay u gaaraan UHC, iyadoo la adeegsanayo madasha adeegga caafimaad oo lama-huraanka/asaasiga ah (Essential Package of Health Services), iyo.

- Fulinta daraasado ku saabsan adeegyada daryeelka caafimaadka aasaasiga ah (PHC) ee heer degmo, oo abuuraya caddeymo cilmi-baaris, si loo diyaariyo xeelado wax ku ool ah oo saameeya xaaladaha maxalliga ah, oo sahlaya in la isdhexgeliyo barnaamijyada caafimaad ee goonida loo fuliyo iyo kuwa ku fula qaabka PHC

\section{Baaxadda iyo nooca qoraallada loo baahanyahay}

SHAJ wuxuu raadinayaa qoraallo mudnaan u leh caafimaadka qaranka, isla markaasna beegsanaya caqabadaha hortaagan nidaamka caafimaadka Soomaaliya, iyagoo keenaya falanqeyn cilmi-baaris iyo talooyin ku saabsan xoojintooda, si ay waxqabadka xagga hore uga galaan. Qorayaasha, gaar ahaan cilmibaareyaasha deegaanka ayaa lagu dhiirrigelinayaa inay soo gudbiyaan:

- Maqaallo assal ah kuwaasoo la jaanqaadaya waxqabadyada mudnaanta leh ee SDGs iyo UHC oo u adeegaya dadka danyarta ah iyo bulshooyinka ay adagtahay in la gaarsiiyo adeegga caafimaad, iyadoo la isticmaalayo cilmi-baaris ku saleysan hab tiro, mid tayo ama hab isku-dhafan.

- Maqaallo Gaagaaban oo la xiriira daraasado tijaabo ah, sahanno goobeedyo ku wajahan deegaanno, waraysiyo ku dhisan habka tayada, iyo caddeymaha cilmi-baarista ee ka dhasha khibradaha maxalliga ah ee soo kordhinaya qiyam iyo aqoon la xiriira hababka fulineed.

- Maqaallo ku wajahan cilmi-baarista lagu dhisayo karti oo diiwaangelinaya sida loo horumariyo kaabayaasha cilmi-baarista ee muhiimka ah iyo sidoo kale xirfadlayaasha caafimaadka guud ahaan, kuwaas oo saldhig u ah u-hawlgalka caafimaadka guud ee dalalka jilicsan.

- Khibradaha isku-xirnaanta oo muujinaya sida iskaashiyadu waxtar ugu leeyihiin dal jilicsan

- Maqaallo waxbarasho oo tilmaamaya habka, hirgelinta iyo qiimeynta dadaallada lagu hagaajinayo aqoonta, iyo heerarka waxqabadka xirfadlayaasha caafimaadka iyo adeegyada ay bixiyaan.

- Maqaallo dib-u-eegid oo ku saabsan horumarinta caafimaadka, ka hortagga cudurrada, ogaanshaha iyo daryeelka cudurrada kuwaas oo ahamiyad weyn u leh caafimaadka bulshada.

- Maqaallada doodda oo lagu wadaagayo arrimo ku saabsan caafimaadka guud iyo siyaasadaha khuseeya dawladaha jilicsan.

- Qoraallada tilmaamaya habraaca hawsha ee khuseeya caafimaadka guud iyo daryeelka 
caafimaad ee ku saleysan caddeymo cilmi-baaris, iyo

- Qoraallada aragtida caafimaadka guud ee soo bandhigaya aqoon ficil ku saleysan iyo qoraallo afkaar ku saleysan, sida daraasado kiisas iyo warar khuseeya caafimaadka guud.

\section{Aragtida iyo himilada}

SHAJ wuxuu gacan ka geysan doonaa kobcinta waxqabadyada caafimaadka guud, iyada oo loo marayo iskaashiga u dhexeeya xubnaha qaybta ka ah joornaalka, wuxuuna sidoo kale sare u qaadi doonaa aqoonta iyo horumarinta kartida. Joornaalka ujeeddadiisu waa inuu fududeeyo hirgelinta ku saleysan caddeymaha cilmibaarista, iyadoo bulshadu noqonayso xudunta go'aan qaadashada iyo hababka isbeddellada. SHAJ wuxuu sidoo kale dhiiri gelin-doonaa cilmi-baarista caafimaad oo ay fuliyaan waaxyo ka baxsan tan caafimaadka, iyagoo wax ka qabanaya "Adeegyada Bulshada ee Saameeya Caafimaadka" (Social Determinants of Health-SDH). Himiladayada ugu weyn waa in aan gaarsiinno oo faafinno caddeymaha cilmi-baarista dib loo hubiyey, si ay saameyn ugu yeeshaan go'aannada daryeelka iyo siyaasadda caafimaadka iyo ku camal-falkooda. Joornaalku wuxuu u adeegi doonaa sidii buundo nabadeed oo kale iyo qaylodhaan dib-u-heshiisiin iyo kalsooni dhisid heer kasta. Jaamacadaha soomaaliyeed ee ka qeybgalaya waxay muujiyeen sida ay uga go'an tahay himilada joornaalka iyo dhisidda hey’ad maxalli ah oo maamusha.

\section{Is-waafajinta aqoonta iyo ku dhaqankeeda}

Aqoonta u tarjumiddeeda ficil waxay ku dhisantaa iskaashi adag oo u dhexeeya nidaamka bixinta adeegga caafimaad, hay'adaha waxbarashada iyo bulshooyinka deegaamada hoose. Khibradda laga helay isu diyaarinta dib u soo nooleynta iskaashiga cilmi-baaris ee jaamacadaha soomaalida iyo kuwa Sweden wuxuu tilmaamayaa iney suurtagal tahay wada shaqeyn ballaaran, caalami ah, sidoo kalena ku saleysan heer qaran [5]. Dadaallada hadda socda ee lagu soo nooleynayo howlaha caafimaadka guud ee Machadka Qaranka ee Caafimaadka Soomaaliyeed (national Institute of Health) ayaa la filayaa inuu hagaajiyo waxqabadka nidaamyada caafimaad iyo awoodda cilmi baaris ee dalka oo dhan. SHAJ wuxuu soo bandhigayaa madal lagu muujiyo xalalka cilmi-baaris ee ku wajahan dhibaatooyinka caafimaadka, iyo in uu kor u qaado wacyiga bulshada ee ku saabsan baahida loo qabo in la yareeyo sinaan la'aanta caafimaadka, iyadoo la raacayo habka iyo xeeladda ah "Dhexgelinta Caafimaadka Siyaasadaha Dalka oo Dhan-Health in All Policies". Markii lagu daro ka-qaybgalka bulshada iyo awoodsiinteeda, habka barnaamijkani wuxuu si wax-ku-ool ah u xallinayaa farqiga $u$ dhexeeya aqoonta iyo ku dhaqankeeda isaga oo wax ka qabanaya "Adeegyada Bulshada ee Saameeya Caafimaadka” (SDH).

\section{Horumarinta sinnaanta jinsiga}

Dhisidda nidaam loogu hawlgalo sinnaanta jinsigu wuxuu suurta-gelinayaa cilmi-baarista caafimaadku iney higsato meeshii ay-mudnayd, iyadoo loo qirayo saameynta ay ku leedahay ku-dhaqanka caafimaadka iyo natiijooyinkiisaa. Soomaaliya tusaha-sinnaan-la'aanta jinsigu wuxuu taaganyahay 0.776 halkii uu ahaan lahaa 1 (2012), taas oo dalka geynaysa meel aad u xun [6]. Cilmi-baaris wax looga qabanayo arrimaha sinnaan-la'aanta jinsiga ee soomaalida ayaa si weyn u wanaajin kara fahamkeenna ku aaddan dhowr dhinac oo saameeya dhisidda nidaamka iyo siyaasadda caafimaadka. Dhisidda nidaamka loogu hawlgalayo Sinnaanta Jinsiga waxaa lagu horumarin doonaa qoraallada SHAJ.

Waxaan u aragnaa joornaalkeennu inuu yahay marxalad cusub oo lagu qeexayo laguna diiwaangelinayo horumarka caafimaadka guud. SHAJ waxay diyaar u tahay inay kaalin firfircoon ka qaadato halganka loogu jiro soo kabashada degdegga ah ee nidaamka caafimaadka Soomaaliya iyo inay gacan ka geysato aqoonsiga iyo wax-ka-qabadka caqabadaha jira iyo kuwa soo if-baxaya.

\section{Khalif Bile Mohamud, Editor-in-Chief}

\section{Tixraacyo}

[1] Dalmar AA, Hussein AS, Walhad SA, Ibrahim AO, Abdi AA, Ali MK et al. Dib-u-dhiska awoodda cilmi-baarista ee waddammada jilicsan: arrinta ku saabsan hindisaha caafimaadka caalamiga ah ee Soomaali - Iswidishka, Glob Health Action 2017. VOL. 10, 1348693. https://doi. org/10.1080/16549716.2017.1348693

[2] Somali-Swedish Action Group oo ka shaqeeya Cilmi baarista Caafimaadka iyo Horumarinta. Bogsiinta nidaamka caafimaadka qalalaasaha shacabka ka dib. Waxqabadka Caafimaadka ee Glob 2015, 8: 27381 -http://dx.doi.org/10.3402/gha.v8.27381

[3] Aden JA, Ahmed HJ, \& Östergren P-O. Waxyaabaha keena iyo sababaha keena dhimashada hooyada ee Degmada Bosaaso ee Soomaaliya. Daraasad dib-u-eegis ah oo ku saabsan 30 kiis iyadoo la adeegsanayo habka loo yaqaan 'Verbal Autopsy'. Waxqabadka Caafimaadka ee Glob, 12: 1, 1672314, DOI: 10.1080 / 16549716.2019.1672314

[4] Abdullahi MF, Stewart Williams J, Sahlèn KG, Bile KM \& Kinsman J. waxyaabaha ka qayb qaadanaya qaadashada tallaalka carruurta ee Degmada Gaalkacayo, Puntland, Soomaaliya. Waxqabadka Caafimaadka ee Glob, 13: 1, 1803543, DOI: 10.1080 / 16549716.2020.1803543

[5] Pellini A, Cabdi DA ,. Saalax G, , Ali HY, Quintin KL, Hassan MA et al. Cilmi baarista Soomaaliya: fursadaha iskaashiga - Warbixinta SIDA, Febraayo 2020

[6] Daahfurka Haweenka Caafimaadka Adduunka (Wgh) Soomaaliya Cutubka: Warbixinta Dhacdada Febraayo, 2019. https://bit.ly/2Hsp1DT 